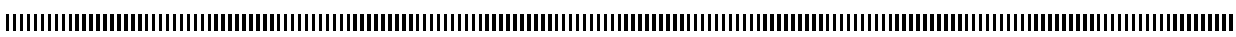

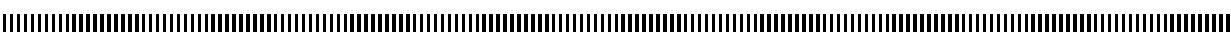
|

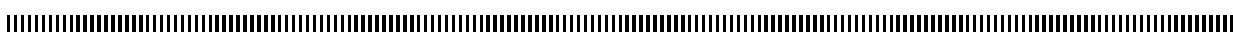

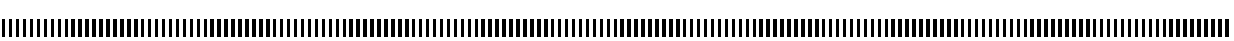
| |

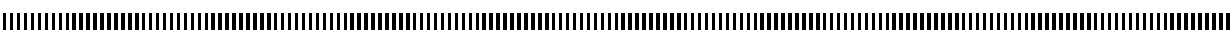
| |

\title{
Management of Low-density Sensor-Actuator Network in a Virtual Architecture
}

\author{
Vianney Kengne Tchendji*, Blaise Paho Nana* \\ * Department of Mathematics and Computer Science \\ Faculty of Science \\ University of Dschang \\ PO Box 67, Dschang-Cameroon \\ vianneykengne@yahoo.fr, blaisepaho@gmail.com
}

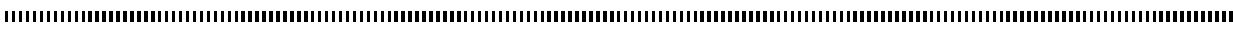

RÉSUMÉ. Les réseaux de capteurs sans fil (RCSF) font face à de nombreux problèmes dans leur mise en œuvre, notamment aux problèmes de connectivité des nœuds, de sécurité, d'économie d'énergie, de tolérance aux pannes, d'interférence, de collision, de routage, etc. Dans ce document, nous considérons un RCSF peu dense, caractérisé par une mauvaise couverture de la zone d'intérêt, et l'architecture virtuel introduite par Wadaa et al qui permet de partitionner efficacement ce type de réseau en clusters. Dans l'optique de router optimalement les informations collectés par chaque capteur jusqu'à une station de base (nœud sink, supposé au centre du réseau), nous proposons une technique d'utilisation des fréquences multiples pour limiter les interférences lors des communications. Ensuite, nous proposons un algorithme de détection de clusters vides permettant d'avoir une vue globale de la répartition réelle des capteurs dans la zone d'intérêt, et ainsi donner la possibilité de réagir en conséquence. Nous proposons également une stratégie de déplacement des capteurs mobiles (actuators) afin de: sauvegarder la connectivité du RCSF, optimiser le routage, économiser l'énergie des capteurs, améliorer la couverture de la zone d'intérêt, etc.

ABSTRACT. Wireless sensor networks (WSN) face many implementation's problems such as connectivity, security, energy saving, fault tolerance, interference, collision, routing problems, etc. In this paper, we consider a low-density WSN where the distribution of the sensors is poor, and the virtual architecture introduced by Wadaa and al which provides a powerful and fast partitioning of the network into a set of clusters. In order to effectively route the information collected by each sensor node to the base station (sink node, located at the center of the network), we propose a technique based on multiple communication frequencies in order to avoid interferences during the communications. Secondly, we propose an empty clusters detection algorithm, allowing to know the area actually covered by the sensors after the deployment, and therefore, giving the possibility to react accordingly. Finally, we also propose a strategy to allow mobile sensors (actuators) to move in order to: save the WSN's connectivity, improve the routing of collected data, save the sensors' energy, improve the coverage of the area of interest, etc.

MOTS-CLÉS : Réseau de capteurs sans fil, architecture virtuelle, déplacement des actuators, cluster vide, évitement de collision(s), connectivité, routage.

KEYWORDS : Wireless sensor network, virtual architecture, moving actuators, empty cluster, collision(s) avoidance, connectivity, routing.

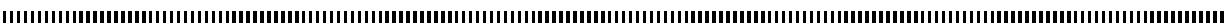




\section{Introduction}

For few years now, many improvements have been made in domains such as microelectro-mechanical systems (MEMS) technology [9], wireless communications, and digital electronics. This enabled the development of micro components that easily combine data collection tools and wireless communication devices, and then opens a wide scope to wireless sensor networks (WSN) $[3,5,8,12]$. Usually called micro-sensors or simply sensors, these devices with limited resources (bandwidth, computing power, available memory, embedded energy, etc.) have revolutionized traditional networks by bringing the idea of developing sensors networks based on the collaborative effort of a large number of sensors operating autonomously, and communicating with each other via short-range transmissions [6, 7]. The vulnerability of the radio communication that sensors are using added to their resource limitations are factors that raise many problems (interference, intrusion, disconnection, data integrity, etc.).

Nowadays, this technology allows sensors to move while remaining connected. When the transmitters are relatively close and use the same frequencies, there may be interferences while sending messages. The messages that never arrive because of collisions can make the network useless. Radio frequencies should be better used to avoid collisions during the various message's broadcast. In addition, it is common to see WSN composed of several thousand units [4]. In these large networks, the sensors can be grouped into clusters based on their proximity in order to significantly increase the scalability, economy of energy, routing, and consequently the lifetime of the network. The structure provided by this partitioning allows the use of various techniques to improve the quality of a WSN, such as data aggregation [11, 12]. One of the biggest challenges of wireless sensor networks remains a fast and energy efficient routing protocols. If in a sensor network, communications are not well organized, the sensors will communicate haphazardly, and spend their energies unnecessarily. This may cause the networks to be quickly off. Similarly, in a real-time application, the data must quickly reach the base station so that decisions can be taken consistently.

Several works have already focused on this field of study. For example Wada and al [1] has set up a virtual architecture which provides a powerful and fast partitioning of the network into a set of clusters, making administration of WSN more easier. S. Faye and J. F. Myoupo [2] were also interested in this topic, showing the first way to route messages in a sparse network. They also showed how to optimize routing using mobile sensors (actuators). Their work has helped to establish a routing protocol that quickly routes the packets to the base station while limiting some redundancies in the clusters. F. Saffre and al [10] were interested in the use of communication channels to limit collisions during the broacastings.

In this paper, we consider a low-density WSN where the distribution of the sensors is poor, and the virtual architecture introduced by Wadaa and al [1]. The latter is created and orchestrated by base station, which is able to split the network into a set of clusters, depending on the strength and direction of the broadcast it can perform. Here, many empty clusters can be formed in the virtual architecture, unlike in [1] where the WSN is assumed to be dense.

In order to effectively route the data collected by each sensor to the base station (sink node, located at the center of the network), we firstly propose a technique using multiple communication channels to greatly reduce collisions during communications. Secondly, we propose an algorithm for the detection of empty clusters, allowing us to know the area 
actually covered by the sensors after the deployment, and therefore, gives the possibility to react accordingly. Finally, we propose a strategy to allow mobile sensors (actuators) to move in order to : save the connectivity of the WSN, improve the routing of collected data, save the energy of our sensors, improve the coverage of the area of interest, reduce the time taken by packets to reach the base station, etc.

The rest of this paper is organized as follows : in section 2, we present the virtual architecture in which we work. Then in section 3, we present our collision(s) avoidance mechanisms. We present a technique of detecting empty clusters in section 4 , followed in section 5 by our method of strengthening strategic points by the actuators, and the technique used to properly move the actuators. In section 6 , we present the overall structure of our fast and energy efficient routing protocol. Examples and simulation results are presented in section 7. Finally, a conclusion ends the paper.

\section{Architecture of the virtual sensor network}

\subsection{Anatomy of a sensor}

This is the basic equipment of any WSN. It has three main tasks : information collection from the deployment area, light treatment (optional) on the collected data and sharing these data with other sensors through multi-hop routing. Despite the great diversity (temperature sensors, humidity, pressure, etc.) existing on the market, they are all mounted on the same architectural diagram mainly made of a unit of : capture, processing, storage, communication, and energy. This material may be supplemented or reduced according to the developer [3]. One can for example add a locating system such as a GPS (Global Positioning System), a mobilizer (to get an actuator). The main and optional elements (represented by dashed lines) are shown in figure 1.

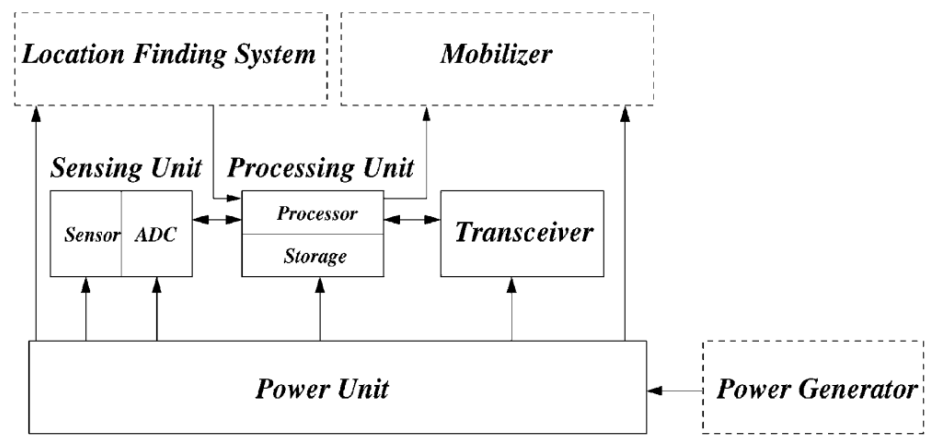

Figure 1 - Hardware architecture of a sensor.

\subsection{Virtual network architecture}

Let's consider a special sensor called the sink or base station (BS) unconstrained by common sensors's limits and capable of omni-directional transmissions at various transmit power that allows it to covers different transmissions radius forming coronas, and directional transmissions at various angles forming angular sectors. Once deployed in the supervised area (figure 2a), the sensors can be grouped in clusters (as described in [1]) 
depending on the corona and the angular sector in which they are located (see figure $2 b$ ). Thus, the intersection of the corona $i$ and the angular sector $j$ forms the cluster $(i, j)$. Exceptionally, the sink is alone in its cluster and its coordinates are $(-1,-1)$. Since the network is sparse, it is important to identify the empty clusters. This allows the sink to have an overview of the area covered by the sensors, and to achieve a better monitoring.

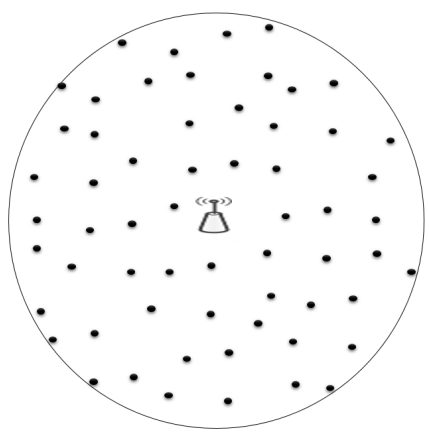

(a) Example of a basic WSN.

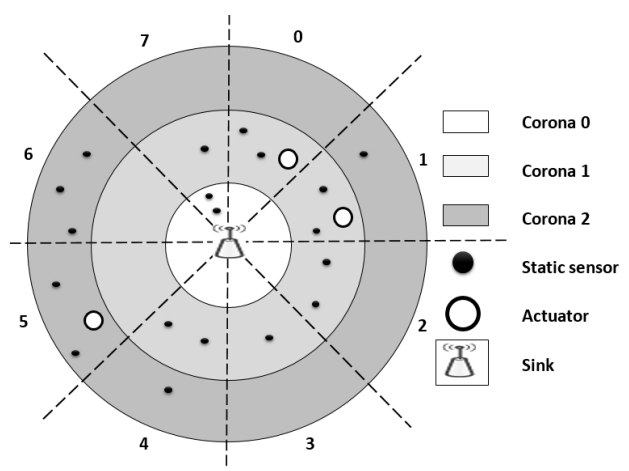

(b) A sparse virtual architecture.

Figure 2 - A WSN represented in a virtual architecture.

For the rest we advance the following hypotheses.

\subsection{Hypotheses}

The network is fully clustered as shown in figure $2 \mathrm{~b}$ using the technique described in [1]. Furthermore,

- Each sensor has a unique identifier $I D$ in the network. The sensors are static and form a connected network. Adding or removing a sensor is a rare event;

- A node is able to estimate its residual energy $E_{r}$ and the sink has the ability to broadcast messages in the network at different radius, or at different angles;

- The time is divided into slots of length $r$. The sensors are aware of the number of coronas and angular sectors. The local clock of each sensor is synchronized with the sink's clock;

- A message sent by a sensor reaches all the sensors located in its transmission range within a slot;

- Clusterisation is made such that all sensors of a given cluster can communicate with each other and some sensors of neighbor clusters.

With these hypotheses we can tackle the first contribution of this paper which is to limit collisions at different message transmissions both in a cluster and amongst clusters.

\section{Collision avoidance mechanisms}

In this section, we show how to avoid interference between the messages exchanged amongst the sensors of the same cluster, and those exchanged between the sensors of neighboring clusters. 


\subsection{Intra cluster collision(s) avoidance : Sharing a communication channel amongst many sensors}

To quickly and correctly route the collected data to the base station (through the cluster-heads), we must prevent messages from collision risks. For this, there are several techniques :

FDMA (Frequency Division Multiple Access) : A communication channel is a frequency range. Each sensor will then have its own sub communication channel. In this case, we will ensure to limit the number of sensors per cluster to ensure that the channel is not divided to the point of being unable to carry sensor's data.

TDMA (Time Division Multiple Access) : Each sensor will then have its own time space, large enough, to transmit its data. Here too, we should make sure we limit the number of sensors per cluster to allow cluster-head to collect data from all stations of their cluster at a time not very long. A good time synchronization that allows each sensor to retrieve his messages and send hers without interfering with other sensor's messages, and in the allotted time is one of the major problems of this method.

CDMA (Code Division Multiple Access) : The allocated frequency range supports its spread spectrum technique. But a problem would remain : the modulation and demodulation operations still require significant computing capacity, which implies more energy and expensive components.

CSMA/CA (Carier Sense Multiple Access with Collision Avoidance) is a variant of the CSMA/CD (Carier Sense Multiple Access with Collision Detection) protocol, which allows the CSMA method to work when collision detection is not possible, as in hertzian networks. Its operating principle is to resolve contention before data is transmitted using acknowledgments and timers. The transmitter tests the channel repeatedly to ensure that there's no activity (i.e. no other station is transmitting). Any received message must be acknowledged immediately by the receiver. Any other new message can be sent only after a certain period to guarantee transport without loss of information. The non-return of an acknowledgment, after a predetermined time, makes it possible to detect if there has been a collision. This strategy not only makes it possible to implement an acknowledgment mechanism at the frame level but also has many advantages such as : it is simple and economical, since it does not require a collision detection circuit unlike to CSMA/CD ; It can be used in wireless sensor networks ; Control of restraint is relatively simple; At low traffic levels, information flow is high; It is not subject to synchronization constraints; It is distributed ; the bandwidth is not divided, which allows fast data transfer; In most of WSN, the collected data is not large, so they are not usually fragmented, and even if that were the case, the CSMA/CA's fragmentation-reassembly mechanism can solve the problem; It enables to share access to the medium, resolves interference and concurrency access problems; his back-off algorithm gives the same probability to the various sensors to access the channel; even if in a given cluster, the informations' harvesting time is relatively short, sensors that were unable to send their data during the allotted time can always do it at the next round thanks to the equiprobability of the back-off algorithm; etc. For these reasons, we choose to use CSMA/CA. 
In summary, CSMA/CA is an appropriate way of solving interference problems and sensor's concurrent channel access in a cluster. But, it is unwise to use this technique over the entire network, because it is possible that sensors in neighboring clusters have adjacent transmission range, and therefore, can not communicate at the same time without risk of collision. To enable them to communicate at the same time, and thus reduce the latency time due to the access time to the communication channel, an additional tip must be associated with the CSMA/CA protocol. To do this, we propose the modulation of frequency of communication between neighboring clusters.

\subsection{Inter clusters collision(s) avoidance : Communication channels distribution}

Our idea is to assign different communication frequencies to neighboring clusters, so that their sensors can communicate at the same time without risk of collision. To do this, we rely on the techniques of graphs coloring.

In graph theory, to color a graph refers to assign a color to each vertex such a way that two vertices joined by an edge have different colors. The frequency assignment in a network is often compared to the graph coloring problem : defining a graph where vertices represent issuers. Two emitters are connected when they are close enough to interfere. Each emitter has a color representing for us, a communication channel. The issuers represent for us the clusters of our virtual architecture. We generally try to minimize the number of colors (channels), said chromatic number while respecting the fact that two related issuers (clusters) should not have the same color (do not transmit on the same channel), but the sensors of the same cluster communicate on the same channel. This minimization of the number of colors (channels) allows the reuse of frequencies.

There are some accurate algorithms for the coloring of a graph using the minimum number of colors such as the Zykov's algorithm $[18,19]$ which uses the branch an bound method; but this class of algorithms have an exponential complexity. Heuristics such as those of Welsh and Powell proposed in [16] and [17] can be used to achieve an acceptable results in polynomial time. An allocation of frequencies to clusters of a sensor network using previous techniques could give the result of figure $3 \mathrm{a}$. But for our use, this frequency distribution also pose interference problems for some clusters. For example, if a sensor $A$ from the cluster $(2,1)$ wants to send a message to the sensors of cluster $(1,1)$, it will listen to the communication channel of the cluster $(1,1)$ (using channel 4) to seek if its channel is free. Note that there's also a neighboring cluster (cluster $(3,1)$ ) communicating on channel 4 . If the channel is indeed free in cluster $(1,1)$ but busy in cluster $(3,1)$ which also uses channel 4, then sensor $A$ will think that the recipient's channel (channel of cluster $(1,1)$ ) is busy (because clusters $(1,1)$ and $(3,1)$ are both using the same channel 4$)$ and will try to postpone the transmission of its message. On the other hand, this inadequacy can cause enormous delays in the delivery of messages which can be very damaging especially for a real-time application.

To overcome this problem, we propose a greedy heuristic (algorithm 1) allocating communication channels at a distance of two hops to different clusters of our virtual architecture. The communication channels are distributed such that two clusters separated by a single intermediate cluster use different channels. This will have for first effect to reduce the flooding of the network, because a message will be confined in a single cluster since it is transmitted using a particular frequency. We will see in the following that it can get out of this cluster only via a gateway node that controls all the outgoing messages. 
The heuristic we propose performs a cluster by cluster processing. For each cluster, it lists its neighbors (including those at two hops), and derives the frequencies used by these neighbors. The channel assigned to the cluster is the smallest unused channel in the vicinity of the cluster.

When the base station has finished determining the communication channels, each cluster is then alerted by a message UseFreq broadcasted in an angular section (or angular wedge) covering the cluster. This message allow the sensors of the recipient cluster to update their communication channels according to algorithm 2 . The message UseFreq consists only of small numbers. The first is a real number defining the amplitude of a channel, the second is an integer representing the index of the frequency that the cluster should use, the third is a list of small integers representing the index-numbers of the frequencies of neighboring clusters and the last is a pair of integers representing the coordinates of the cluster concerned. These index-numbers allow a sensor to calculate the frequency range on which to transmit a message towards its own cluster and also to its neighboring clusters.

Figure $3 \mathrm{~b}$ shows an allocation of frequencies in two hops using our algorithm. If we look carefully, we can realize that the sensors of the first corona are not subjected to the constraint of distancing the channels by two hops, since they transmit their messages directly to the sink. On the other hand, when the sink wants to communicate with a sensor, it transmits on the frequency of the cluster in which the sensor is located, in the corresponding sector, with a power capable to reach the cluster. However, communication is very rare in this direction, since almost all messages travel from the sensors to the base station.

We also use the CSMA/CA protocol for inter-cluster communication for its many benefits. Each cluster has a leader node also called cluster-head $(\mathrm{CH})$ which can both play the role of a common sensor and the role of the gateway by wich a message can go out of the cluster. Each cluster (apart from those of the first corona) also have an other cluster called relay cluster or cluster-relay through which passes any message it sends to the sink. When a cluster-head wants to transmit data to the sink, it transmits it on its relay cluster's channel using the back-off algorithm of the CSMA/CA protocol.

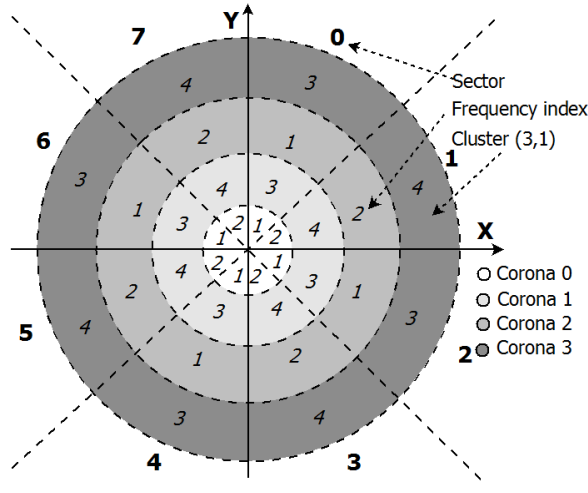

(a) One hop frequency allocation scheme.

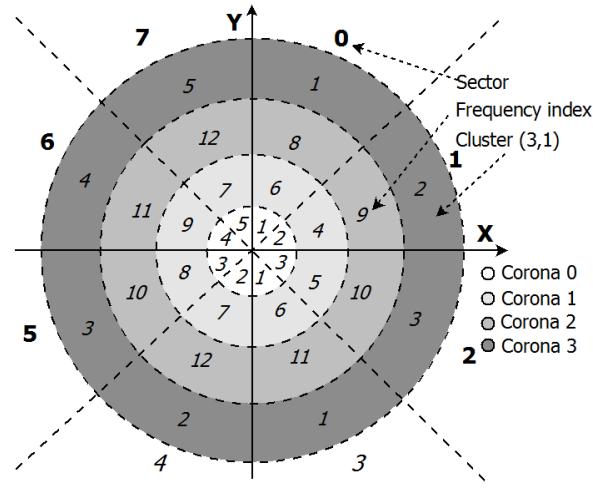

(b) Two hops frequency allocation scheme.

Figure 3 - Frequency allocation scheme. 
Theorem 1 This communication channels allocation algorithm determine a distribution, limiting interference with neighboring clusters in $O(n \log (n))$ time for $n$ clusters.

Proof. In fact, this algorithm is articulated in three main axes. We start by performing a sorting; We know that this operation has a complexity in $O(n \log (n))$. Then comes two loops : the first calculates for the $n$ clusters of the architecture, the frequency to be used; hence a complexity of $O(n)$ for this loop. Then the second makes $n$ broadcast to inform each cluster of the channel to be used; Hence a complexity in $O(n)$ for this second loop. This algorithm thus has an overall complexity in $O(n \log (n))+O(n)+O(n)=$ $O(n \log (n))$.

The available frequency range is divided into $N$ channels and dispatched for each cluster using the directional transmissions reaching the concerned cluster. Each cluster has at must 24 neighbors (including those at two hops). After repeating the algorithm several times on various samples, we noticed that the frequencies range is beeing subdivided into $N=9$ channels for an odd number of angular sectors and $N=12$ channels for an even number of angular sectors.

Now that we know how to avoid collisions at various emissions, we can tackle the next goal of this paper : How to correctly and quickly route messages to the base station. For this, we will start with the detection of empty clusters, and then we will see how to move mobile sensors into these empty clusters to improve the routing.

\section{Detection of empty clusters and distributed cluster-heads election}

This detection of empty clusters phase must precede routing of data in order to define the best path that data should follow to quickly reach the sink (using for example the message propagation tree of figure 5b). It also allows to know the area actually covered by the sensors after the deployment, and therefore, gives the possibility of react accordingly. A cluster is considered empty if it contains no sensor, or if it contains a set of sensors disconnected from the rest of the network (or disconnected from the sink).

For $c$ coronas and $s$ angular sectors, the sink counts $(c \times s)$ clusters in its virtual architecture; therefore, for each message received, it regularly updates two tables $h(c, s)$ and $\operatorname{relay}(c, s)$. Each entry $(i, j)$ of the table $h(c, s)$ contains 1 if cluster $(i, j)$ is not empty and 0 otherwise, allowing the sink to get an overview of the sensors distribution; and each entry $(i, j)$ of the table relay $(c, s)$ contains the coordinates of the relay cluster of the cluster $(i, j)$.

\subsection{Sink's algorithm}

After the partitioning and distribution of communication channels, the sink periodically broadcasts the date on which the detection algorithm will begin. All sensors are awake and the sink initiates the detection. The sink then transmits on each channel of its neighboring clusters (those of the first corona) a message Detect containing its coordinates $(-1,-1)$ and an integer numberOfHops initialized at 0 representing the distance (in terms of number of hops) to the sink (algorithm 3). After this, it wait some acknowledgments ( $A C K$ messages) that will allow it to update the tables $h$ and relay. Due to network connectivity, it is certain that at least one sensor of the first corona will receive this message. During the algorithm, each $A C K$ message transmitted by a sensor towards the sink contains the coordinates of its cluster and those of his relay cluster. Table $h(c, s)$ 
is initialized to 0 . At each reception of a message from a sensor of the cluster $(i, j)$, BS puts 1 in $h(i, j)$ and, in the entry relay $(i, j)$, it assigns the value contained in the variable relay of the received message. The first $A C K$ message arrives 6 slots after the launch of the algorithm (as we shall see later, 1 slot for the transmission of the Detect message, 2 slots for the two timers, 2 slots for the transmission of the messages Head 1 and Head2, 1 slot for the transmission of the $A C K$ message), and then an $A C K$ message arrives every 2 slots; the sink knows that the detection (algorithm 3 ) has ended when it receives no $A C K$ message after more than two slots.

\subsection{Sensor's algorithm}

The network is supposed connected, so for all cluster $(i, j)$ considered non-empty, there is always a path from it to the sink node. Isolated clusters cannot reach the sink and are considered empty even if there are not. There are five main events in the detection of empty clusters :

1) The reception of a message Detect asking sensors to indicate their coordinates ;

2) The reception of a message Detect_timer asking sensors to be ready for the election of the cluster-head;

3) The reception of a message Headl sent by a node to propose itself as a gateway node (or cluster-head), if it has the smallest distance to the sink;

4) The reception of a message Head2 sent by a node to notify the other sensors of his cluster that he is the cluster-head and ;

5) The reception of a message $A C K$ sent by a cluster-head to the sink node to indicate their coordinates, and also the fact that the cluster is not empty.

\section{Reception of a message Detect}

When a sensor receives a Detect message, it checks two conditions : if it has not already had to route a Detect message, and if its cluster is adjacent to that of the sender of this message. The reason is the following : firstly, each sensor has to route only a single Detect message, because any other similar message necessarily comes from a more distant cluster to the sink. Secondly, the sizes of the clusters of the lower coronas are smaller than the sizes of the more distant clusters, since the sensors transmission range are constant, in lower coronas, transmission can easily cover several neighbors clusters. If one of the two conditions is not satisfied, it ignores the Detect message. Otherwise, if it is the first time he receives the Detect message (i.e. if it has not received a Detect_timer message), it builds a message Detect_timer containing the starting date of the distributed cluster-head election noted End_Date, and broadcasts it on its own cluster's channel. Any sensor that receives a Detect_timer message can't send an other Detect_timer message. It just waits still the date End_Date to begin the cluster-head's election algorithm.

While waiting for End_Date, sensors that receive the Detect message (including the one that received the first Detect message), compare the value Detect.numberOfHops to their local value number Of Hops. If Detect.numberOf Hops $+1<$ numberOf Hops this means that the message comes from a nearest cluster from the sink (numberOf Hops is initialized at $\infty$ for all the sensors, forcing the sensors to route a single Detect message), then the sensor puts its variable numberOf Hops at Detect.numberOf Hops + 1 , takes the sender as relay cluster, builds an other Detect message an broadcast it to permit other sensors to identify theirselves (algorithm 4). 
At the time End_Date contained in the Detect_timer message previously received, the distributed cluster-head election algorithm (algorithm 6) is immediately executed. At the end of this algorithm, the elected cluster-head sends an $A C K$ message to the sink using the frequency of its relay cluster.

Before the distributed cluster-head's election in each cluster, every sensor of the given cluster that are able to join some relay cluster have received a Detect message from this relay cluster, and know how far they are from the sink (numberOf Hops); they are also able to estimate their residual energy $E_{r}$. We rely on these information available locally at each sensor to elect the cluster-head. The algorithm takes place in two phases, requiring only two broadcasts (messages Head1 and Head2). Each sensor with a residual energy superior or equal to the energy threshold $E_{s}$ begins by calculating a first timer at the end of which it is supposed to broadcast a Head1 message to propose itself as the leader. The local channel is normally free and the timer is calculated such that it is the nearest sensor to the sink that will access the channel. If there are many sensors having the same distance to the sink, the one with the highest $I D$ accesses the channel. The duration of this first timer is $\left(1-\frac{1}{\text { numberOfHops }}+\frac{e^{(-I D)}}{\Lambda}\right)$ slots ${ }^{1}$. The first sensor accessing the channel broadcasts its Head1 message and oblige other sensors to cancel the sending of their Head1 message (algorithm 7 : Reception of a Head1 message).

\section{Reception of a message Head1}

The sensor that sent the Head1 message, and other sensors that have received the message $H e a d 1$, and which have a residual energy greater than the threshold $E_{s}$, and have a numberOfHops less or equal to the one contained in the message Head1, calculate and arm a second timer. This second timer rely on the residual energy $E_{r}$ of each sensor, and is such that it is the sensor having the highest residual energy that accesses the channel the first. The duration of this second timer is $\left(\frac{1}{E_{r}}+\frac{e^{(-I D)}}{\Lambda}\right)$ slots. At the end of this second timer, a single sensor (this is actually the cluster-head) access the channel and notifies (by sending a message Head2) all other sensors of its cluster that it is now the cluster-head.

\section{Reception of a message Head 2}

At the reception of the message $\mathrm{Head2}$, the receiving sensor disarms the second timer and puts its local data up to date (algorithm 8 : Reception of a message Head2). This message contains the ID of the cluster-head, the coordinates of the relay cluster, as well as the distance (in terms of number of hops) of the cluster to the sink. The cluster-head is charged of building and sending an acknowledgment ( $A C K$ message) to the sink. The cluster-heads of the first corona send their $A C K$ message directly to sink, while those of the other coronas send their $A C K$ message through the relay cluster.

\section{Reception of a message $A C K$}

A sensor that receives a message $A C K$ from the neighbor node checks whether this message is for its cluster. In this case, it sends it to its gateway node which checks if it has not already routed an $A C K$ message from the same cluster. In this case, it broadcasts it on the channel of its relay cluster. Otherwise, it simply ignores the message.

Theorem 2 For c coronas and s angular sectors, the empty clusters detection algorithm requires $[c \times(s-1)+4]$ slots in the worst case. 
Proof. The worst case corresponds to the one where the Detect and $A C K$ messages move from the sink to the furthest cluster following a path in the form of a spiral as in the figure 4. For $c$ coronas and $s$ angular sectors, the Detect message needs $\left(\frac{c}{2} \times(s-1)\right)$ slots to reach the most distant sensor if $c$ is even, $\left(\frac{c-1}{2} \times(s-1)+1\right)$ slots if $c$ is odd, and 1 slot if $c=1$. The $A C K$ message sent by this sensor takes an equivalent amount of time to reach the sink. Additionally, it is easy to see that the election of a cluster-head takes 4 slots in each cluster. So the first $A C K$ message arrives 6 slots after the launch, then another arrives every 2 slots, and the last one arrives $[c \times(s-1)+4]$ slots after the launch.

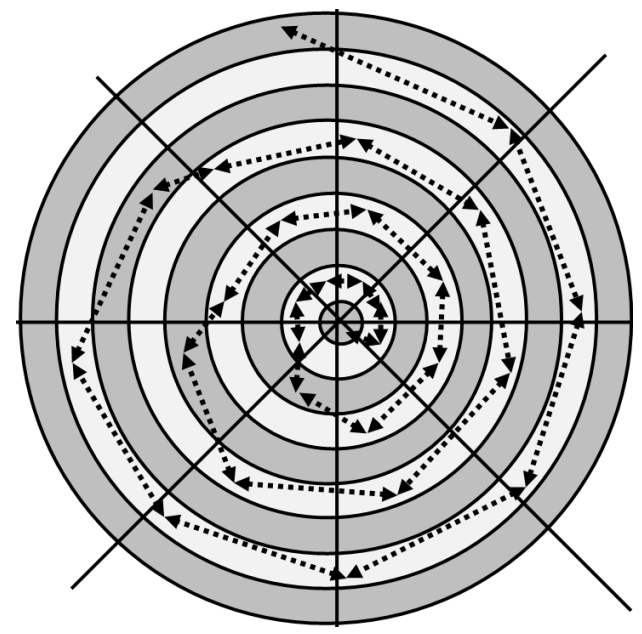

Figure 4 - Longest path traveled by a message to reach the sink node.

After the detection of empty clusters and the election of cluster-heads, the network is ready to be use. Sensors can collect data and send them to the sink through possibly non optimal paths. The following section aims to optimize routing by offering more optimal paths.

\section{Routing optimization using mobile sensors}

This section is inspired from [2]. Here we introduce the actuators : sensors with a mobilizer, allowing them to move on the sink's order. They can be used for many purposes, depending on the user, for example :

- Being the $\mathrm{CH}$ in a cluster where the residual energies of the sensors are under the threshold energy ;

- Collect and route information in isolated areas ;

- Connect a sub isolated connected network to the main network;

- Being sent in strategic empty clusters (purpose of this paper) to optimize the routing.

\subsection{Searching and filling strategic empty clusters by actuators}

From the table $h(c, s)$, the sink can identify empty clusters. In order to know which ones it is going to fill first, it should produce the messages spreading tree like in figure $5 \mathrm{~b}$ 
by using the two tables it has like this : Take BS as root and the first tree's leaf. As long as there's an unvisited leaf $(i, j)$, search in the table relay $(c, s)$ the cluster that has the cluster $(i, j)$ as relay cluster and add them as the sons of $(i, j)$.

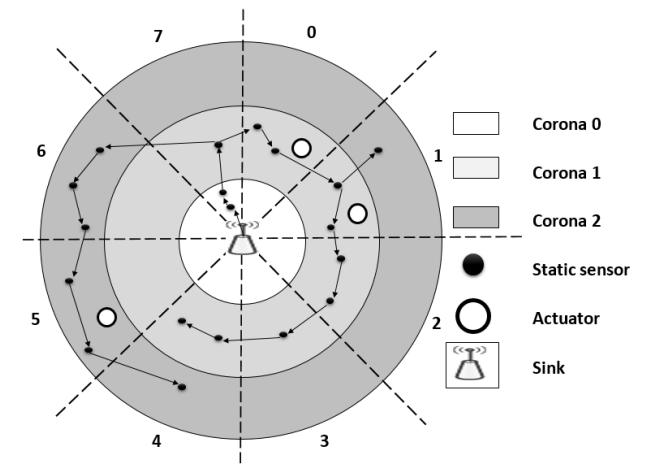

(a) Propagation of messages in the supervised area.

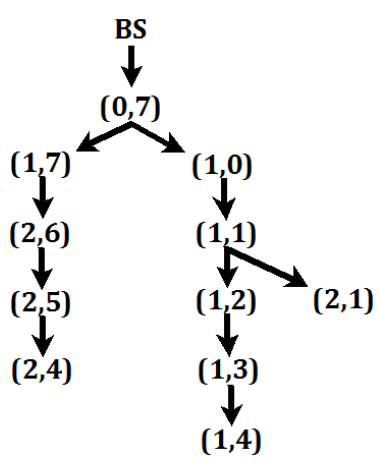

(b) Messages spreading tree.

Figure 5 - The messages spreading tree obtained after the detection of the empty clusters.

Filling a strategic empty cluster has the effect of reducing the tree's height (figure $5 b$ ). The ideal one would be to reduce this height to the number of coronas of the virtual architecture. The routing will be optimal if for any cluster of corona $k$, the transmission of a message towards BS passes through $k$ intermediate clusters.

\subsubsection{Rule of detection of clusters which access can be improved}

To optimize the routing to a cluster, it would be good to know whether the current access is optimizable. In figure 5 , it is the case of cluster $(1,4)$ which is at 5 intermediate clusters from BS instead of 1 if an actuator were judiciously placed in cluster $(0,3)$.

Rule : Let $\mathbb{T}$ be a messages spreading tree similar to that of figure $5 \mathrm{~b}, \operatorname{prof}(i, j)$ denotes the depth of the cluster $(i, j)$ in the tree $\mathbb{T}$. The path from sink to $(i, j)$ can be improved if there is another cluster $\left(i^{\prime}, j^{\prime}\right)$ with a depth $\operatorname{prof}\left(i^{\prime}, j^{\prime}\right)$, such as $i^{\prime} \geq i$ and prof $\left(i^{\prime}, j^{\prime}\right)<\operatorname{prof}(i, j)$, i.e. $\left(i^{\prime}, j^{\prime}\right)$ is in a corona greater or equal to $(i, j)$ 's but in the tree $\mathbb{T},\left(i^{\prime}, j^{\prime}\right)$ appears at a depth less than $(i, j)$ 's.

\subsubsection{Detection of strategic empty cluster to fill in priority}

To determine this priority cluster (PC), we establish for every corona $a$ the list $L[a]$ of clusters of this corona which access can be improved (i.e for $C$ coronas, we should have $C$ lists). Each list $L[a]$ contains the coordinates $(a, j)$ of clusters of the corona $a$ which access can be improved. It is in the form : $L[a]=\left[\left(a, j_{1}\right),\left(a, j_{2}\right), \ldots,\left(a, j_{n}\right)\right]$. From each list $L[a]$, we extract the longest list $L_{s}[a]$ made of consecutive clusters of $L[a]$. In the list $L_{s}[a]$, each $(a, j)$ represents the coordinates of the clusters of corona $a$ that follows in the message spreading tree. The coordinates $(x, y)$ of PC are deduced from the longest sub list $L_{s p}[a]$ taken among the $L_{s}[a]$ extracted lists. $x=a-1$ and $y$ is equals to the default rounding average of $j$ (the $j$ are the second components items $(a, j)$ of the list $L_{s p}[a]$ ). As long as there are available actuators, it is necessary to move an actuator at the cluster $(x, y)$, another at the cluster $(x-1, y)$ if it is empty, $\ldots$ another at the cluster $(0, y)$ if it is empty. The process can be repeated until the routing is optimal, i.e. up to $\operatorname{prof}(\mathbb{T}) \leq C$. For the example of figure 5, the determination of $\mathrm{PC}$ is presented in annex A. 
Before moving a mobile sensors, the sink node must calculate the distances from it to the target empty cluster, it is better to choose the most appropriate actuator, based on distance, residual energy, availability, etc.

\subsection{Moving a mobile sensor}

We propose to move an actuator from the cluster $(x, y)$ to the empty cluster $(j, k)$. To properly move our actuator from cluster $(x, y)$ to cluster $(j, k)$, it will need a distance (not in terms of number of hops) and a direction. To simplify our calculations, we should be in a Cartesian plane. For this we describe here how to transform our current coordinate system (Dynamic Coordinate System : DCS) in a Polar Coordinate System (PCS) and then in to a Cartesian Coordinate System (CCS).

\subsubsection{Correspondence between the DCS, the PCS and the CCS}

To get the distance and the direction to follow, we must define a reference. Thus the origin of our reference will be the sink. The $Y$ axis is taken such that it coincides with the left edge of the first section (section 0). The $X$ axis is at a quarter turn from the $Y$ axis so that the angle $X, \widehat{\operatorname{sink}}, Y$ is direct (figure 6a). Any point of the DCS is discoverable using $p$ (its distance from the sink) and $\varphi$ (angle measured from the $Y$ axis) as in figure $6 \mathrm{~b}$.

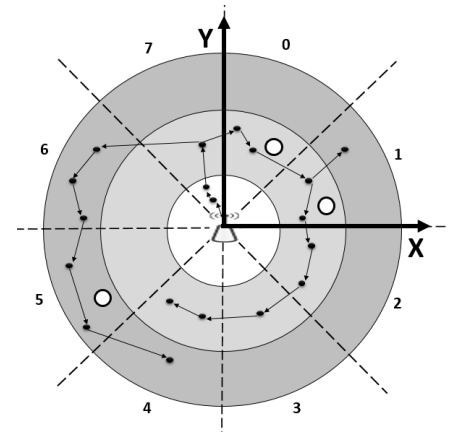

(a) Definition of the reference

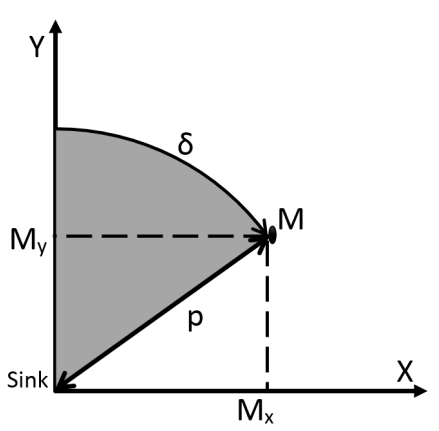

(b) PCS and CCS

Figure 6 - Correspondence between the DCS, the PCS and the CCS

Denote ' $\alpha$ ' the angle of a sector and ' $e$ ' a thickness of a corona, we can state corollary 1.

Corollary 1 Let $M$ be a sensor of the cluster $(c, s)$ assumed at it center. In the PCS, $M$ has the coordinates $(p, \varphi)$ where $p=c \times e+\frac{e}{2}$ and $\varphi=s \times \alpha+\frac{\alpha}{2}$; Let $M(p, \varphi)$ be a point in the PCS. In the CCS, $M$ has coordinates $(x, y)$ with $x=M_{x}=p \sin (\varphi)$ and $y=M_{y}=p \cos (\varphi)$.

Now we can start the necessary calculations (distance $p$ and angles $\varphi$ ) to move the actuators.

\subsubsection{Calculation of the distance $(p)$}

The distance between two points $A$ and $B$ of the plane is given by the norm of the vector $\overrightarrow{A B}$, denoted $\|\overrightarrow{A B}\|$ or just $A B$. According to figure $6 \mathrm{~b}$, an actuator that moves from the sink node (with coordinates $(0,0)$ ) to the point $\mathbf{M}$ (with coordinates $(x, y)$ ) must cover the distance $p=\|\overrightarrow{\operatorname{sink} M}\|=\|(x-0, y-0)\|=\|(x, y)\|$. But $\|\overrightarrow{\operatorname{sink} M}\|^{2}=$ 
$\left(\operatorname{sink} M_{x}\right)^{2}+\left(\sin k M_{y}\right)^{2}=x^{2}+y^{2}$. Thus $\|\overrightarrow{\operatorname{sink} M}\|=\sqrt{x^{2}+y^{2}}$. So we have corollary 2 .

Corollary 2 Moving an actuator from the center of the cluster $A\left(c_{1}, s_{1}\right)$ of polar coordinates $\left(p_{1}, \varphi_{1}\right)$ to the center of the cluster $B\left(c_{2}, s_{2}\right)$ of polar coordinates $\left(p_{2}, \varphi_{2}\right)$ returns to move this actuator from the point $A\left(A_{x}, A_{y}\right)$ to the point $B\left(B_{x}, B_{y}\right)$ of the cartesian coordinates system on a distance $p=\|\overrightarrow{A B}\|$ where $\overrightarrow{A B}\left(B_{x}-A_{x}, B_{y}-A_{y}\right)$, $p=\|\overrightarrow{A B}\|=\sqrt[2]{\left(B_{x}-A_{x}\right)^{2}+\left(B_{y}-A_{y}\right)^{2}}, A_{x}=p_{1} \cos \left(\varphi_{1}\right), A_{y}=p_{1} \sin \left(\varphi_{1}\right)$, $B_{x}=p_{2} \cos \left(\varphi_{2}\right)$ and $B_{y}=p_{2} \sin \left(\varphi_{2}\right)$.

\subsubsection{Calculation of the angle $(\varphi)$}

To facilitate the calculation of the value of $\varphi$, let's make a change of reference.

Change of reference : We want to move a mobile sensor from a point $A$ to a point $B$. For this, we define a new reference in which the base vectors are collinear with those of the previous (see figure 7a).

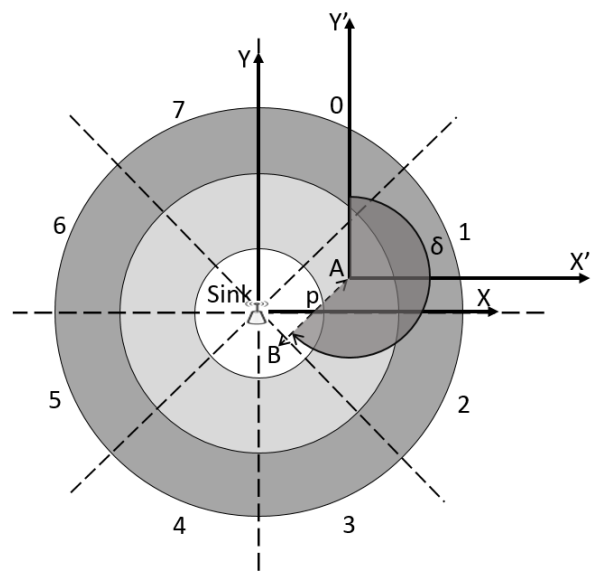

(a) Reference change

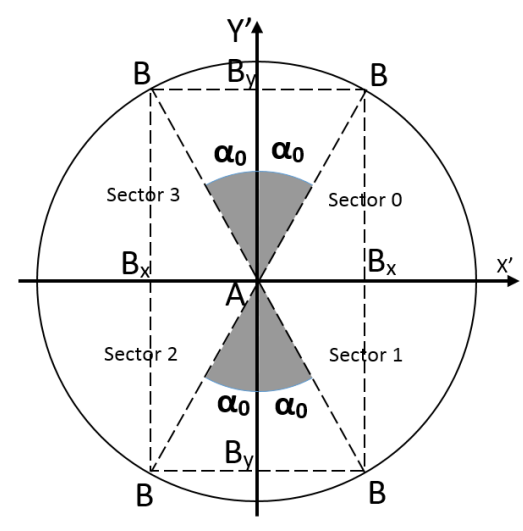

(b) Angle between the axis $Y^{\prime}$ and the displacement vector

Figure 7 - Angle displacement

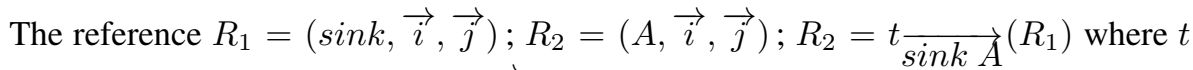
denotes the translation of vector $\overrightarrow{\operatorname{sink} A}$. The coordinates of two points $M(x, y) \in R_{1}$, and $M^{\prime}\left(x^{\prime}, y^{\prime}\right) \in R_{2}$ such that $M^{\prime}=t \underset{\operatorname{sink} A}{(M)}$ are now linked by the following relations : $x^{\prime}=x-x_{A}$ and $y^{\prime}=y-y_{A}$.

Basis vectors of these two references are pairwise collinear, that's why the angles found in one of the references will be equivalent in the second.

Calculation of the inclination $\alpha_{0}$ formed by the displacement vector and the $Y^{\prime}$ axis : The displacement angle $\varphi$ that we want to calculate is strongly related to the angle $\alpha_{0}$ formed by the vector $\overrightarrow{A B}$ and the $Y^{\prime}$ axis. Figure $7 \mathrm{~b}$ presents the different situations we may encounter. We deduced that $\sin \left(\alpha_{0}\right)=\frac{\left|B_{x}\right|}{A B} \Rightarrow \alpha_{0}=\sin ^{-1}\left(\frac{\left|B_{x}\right|}{p}\right)$ where $p=A B$. 
Determination of the displacement angle $\varphi$ : From figure $7 \mathrm{~b}$, point $B$ can be found in one of the four sectors.

Corollary 3 The displacement angle $\varphi$ of an actuator from the point $A\left(A_{x}, A_{y}\right)$ to the point $B\left(B_{x}, B_{y}\right)$ of $(A, \vec{i}, \vec{j})$ reference is given by:

1) if $B$ is in the sector 0 , i.e. $B_{x}>0$ and $B_{y} \geq 0$ then $\varphi=\alpha_{0}$

2) if $B$ is in the sector 1, i.e. $B_{x} \geq 0$ and $B_{y}<0$ then $\varphi=\pi-\alpha_{0}$

3) if $B$ is in the sector 2, i.e. $B_{x}<0$ and $B_{y} \leq 0$ then $\varphi=\pi+\alpha_{0}$

4) if $B$ is in the sector 3 , i.e. $B_{x} \leq 0$ and $B_{y}>0$ then $\varphi=2 \pi-\alpha_{0}$

For a practical example of moving a sensor, see annex B.

Now that we have all the different modules of our protocol, we can now describe how it works.

\section{Unrolling of the routing protocol}

Once the sensors are randomly deployed around the sink, the base station performs the clustering of the deployment area in small clusters easily manageable. Note that at this phase, sensors does not communicate, therefore their energy consumption is quite insignificant. Then the base station determines and allocates communication channels to different clusters to limit inter-clusters interferences. Then comes the empty cluster detection phase initiated by the sink. This step allows the base station to get an overview of the distribution of the sensors in the area of interest. Cluster-heads and relay clusters are also defined at this step. Already at this stage, the routing of collected data can begin. The informations collected in the empty clusters detection phase will allow the sink to move the actuators in strategic empty clusters in oder to optimize the routing.

Once the data are captured, it would be wise to send them as soon as possible to the base station while avoiding network congestion. The messages spreading tree seen previously may be very useful here. This tree optimized by the sink with actuators, allows each cluster to send its data to the sink by the shortest path (number of hops).

In addition, the CSMA/CA protocol executed by each sensor in each cluster and between clusters helps to avoid network congestion. Another way of making the best results would be to attach an aggregation protocol to limit redundant messages routed in each cluster.

\section{Simulation and analysis of our solution}

\subsection{Tools and simulation environment}

Using an HP computer Intel (R) Core (TM) i7-2630QM CPU @2.00 GHz $\times 8,8 \mathrm{~GB}$ of RAM, running Windows 8 Professional; a discrete event network simulator J-Sim; and a sample of 1000 sensors randomly deployed within $10 \mathrm{~km}$ of the sink; we also have a sufficient number of mobile sensors. To remain in the logic of low density network, we have limited number of mobile sensors to $50 \%$ of the number of clusters i.e. 40 mobile sensors for our tests, knowing that few of them will be useful and the rest will serve for possible replacements. The energy threshold is set at $40 \%$ of the total energy of each sensor; the virtual architecture has 10 coronas and 8 sectors of $45^{\circ}$ each. We repeated our tests many times and in what follows we are using the averages of the obtained results. The energy 
model is the one adopted by many efficient contributions [14] $: E=E_{\text {trans }}+E_{\text {recep }}$. $E_{\text {trans }}$ and $E_{\text {recep }}$ are respectively the total energy used for transmissions in the network and receptions, knowing that each sensor has a range of 500 meters, an initial energy of 100 joules, and needs $35.28 \times 10^{-3}$ joule per transmission and $31.32 \times 10^{-3}$ joule per reception. Each actuator has an initial energy of 250 joules and consumes $0.5 \times 10^{-3}$ joule per meter to move. A slot takes $780 \mu \mathrm{s}$. The curves were made with version 5.0 of gnuplot software.

\subsection{Analysis of the simulation results}

\section{Time taken for the election of $\mathrm{CH}$}

For this particular test, we also wanted to test the scalability of our cluster-head election algorithm. For this we carried out the test on an architecture made of 16 sectors and 32 coronas i.e. 512 clusters. On figure 8, each point represents the duration of the cluster-head election in a given cluster. The figure shows that our distributed cluster-head election algorithm which needs only two broadcasts in each cluster stays less than four slots in each cluster.

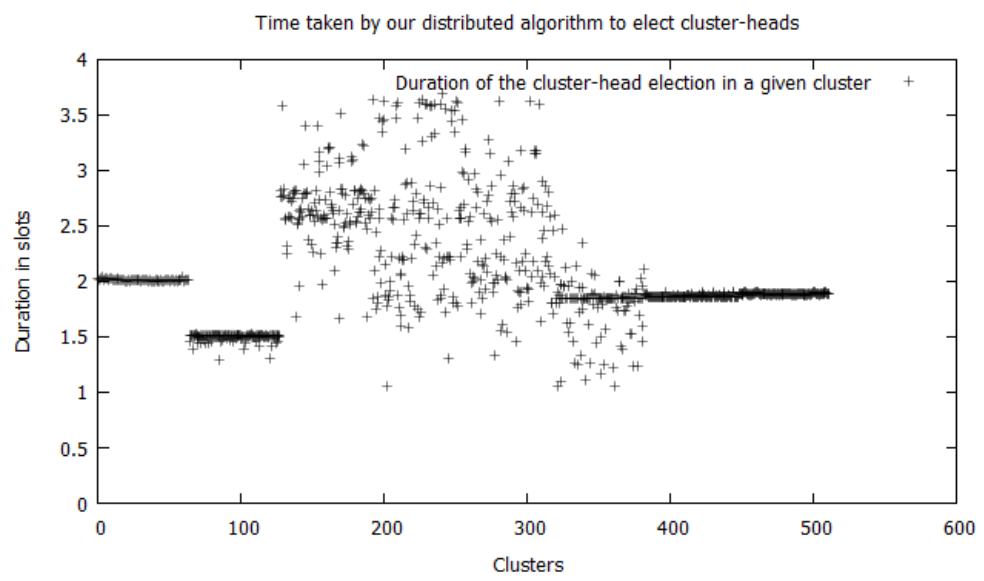

Figure 8 - Time taken for our distributed cluster-head election algorithm in each cluster.

\section{Energy progress}

Figure 9 shows the energy consumption among both ordinary sensors and clusterheads sensors. The sharp fall of energy observed at the beginning of the curve is normal. Initially, $\mathrm{CH}$ are not yet elected, and this operation wastes enough energy. This drop of energy is significantly reduced once the $\mathrm{CH}$ are elected.

\section{Use of actuators to improve energy consumption}

Figure 10 compares the energy consumption of the cluster-heads and ordinary sensors when the routing is not optimized and when routing is optimized with actuators. An economy of energy is observed among both ordinary sensors and cluster-heads sensors. This increases the longevity of the network. The simulation is made for the detection of empty clusters, the election of cluster-head and routing. Here too, the great loss of energy observed at the beginning of the curve is due to the fact that the cluster-head are not elected at 


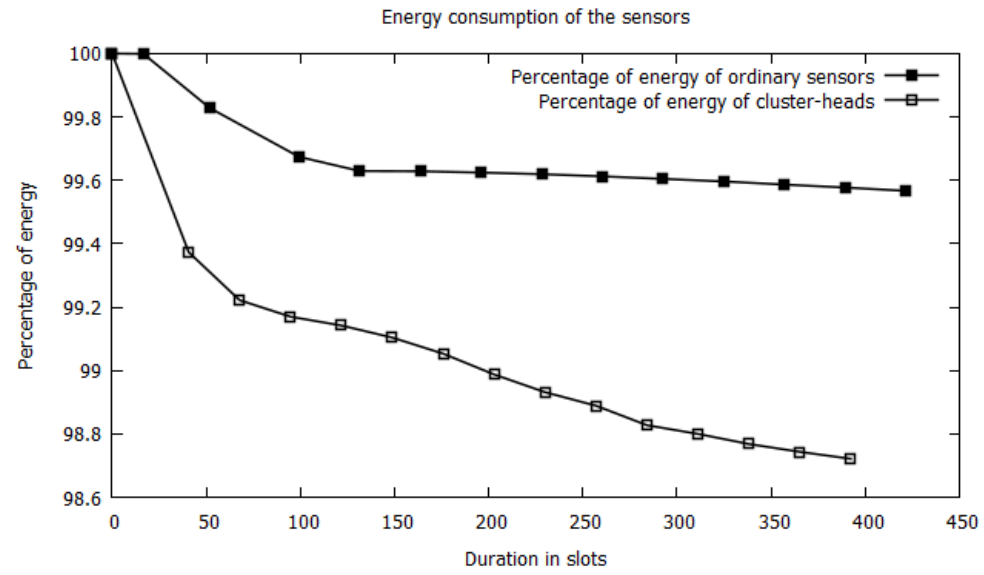

Figure 9 - Energy consumption of ordinary sensors and cluster-heads the beginning. It is clear that this energy loss is significantly reduced once the cluster-head are elected.

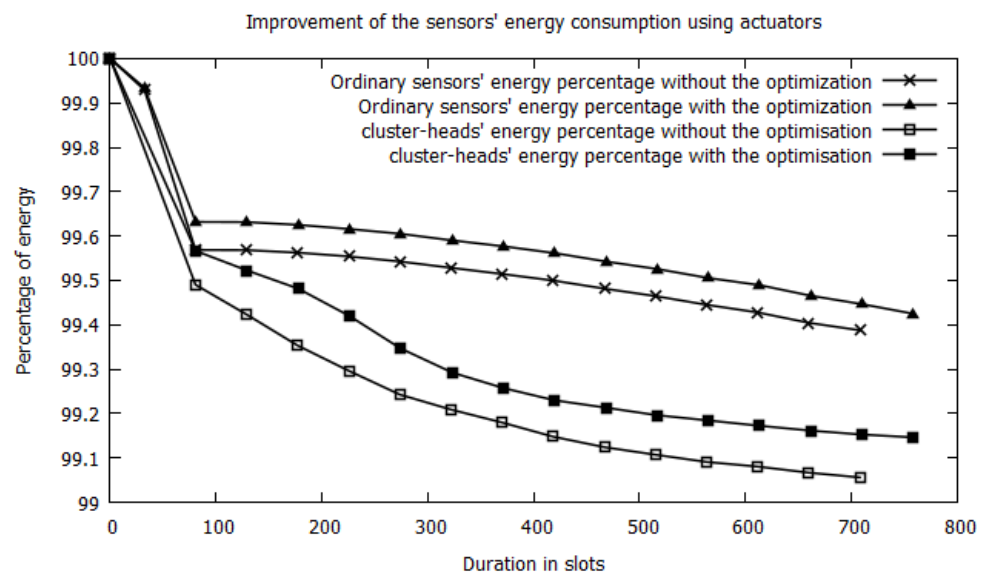

Figure 10 - Improved of the power consumption using actuators

\section{Effect of the number of actuators}

Figure 11 shows that, compared to when actuators are not used, the more we have available mobile sensors, the more routing tends to be optimal allowing enormous gains in terms of energy and messages delivery delay. Also notice that when the routing is optimal, the energy gain and the message delivery delay are also optimized. At that time, it is no longer necessary to continue increasing the number of mobile sensors. The rest of mobile sensors can then be used to replace those that are defective. 


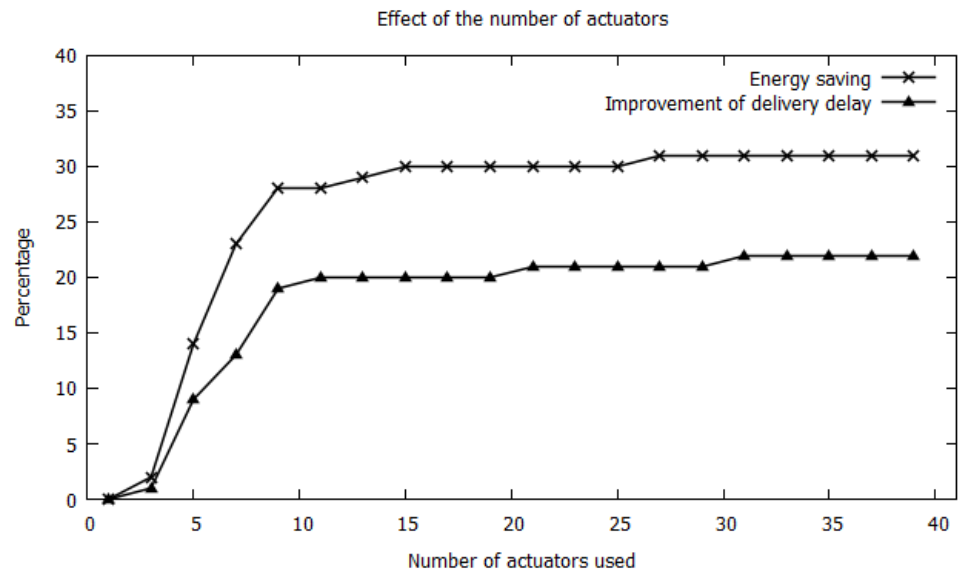

\section{Conclusion}

Figure 11 - Effect of the number of actuators.

In this paper we have presented a virtual architecture that facilitates the management of WSN. We have presented some technics which allows us to avoid collisions in a WSN both between clusters an inside clusters. We also introduced the gateways nodes, their economical election protocol needs only two message transmissions, we show how to limit redundant messages through them. But our main aim was to optimize the routing of collected data towards the sink. That's why we describe a method of detecting and filling strategic empty clusters in which we can send mobile sensors (actuators) in oder to shorten the road taken by messages. We also present other possible utilities of the actuators, and show a new way to perform their movements to improve the routing of the collected data.

In a very soon future, we intend to work on the mechanism of reelection of the clusterhead in a cluster; the mechanism of changing relay cluster if the current relay cluster is no longer accessible and the mechanism of redirection of packets after the positioning of a mobile sensor. It would be also interesting to integrate data aggregation techniques to minimize the number of messages circulating in the network. Authentication and security are also important issues in this kind of network.

\section{Bibliographie}

[1] A. WadaA, S. Olariu, L. Wilson, M. Eltoweissy, K. Jones, « Training a wireless sensor network », Mobile Networks and Applications, Vol. 10, $\mathrm{N}^{\mathrm{o}}$ 1-2, p. 151-168, 2005.

[2] Sébastien Faye, JeAn-Frédéric Myoupo, «Deployment and Management of Sparse Sensor-Actuator Network in a Virtual Architecture », International Journal of Advanced Computer Science, Vol. 2, $\mathrm{N}^{\mathrm{O}}$ 12, December, 2012.

[3] I. F. Akyildiz, Weilian Su, Y. Sankarasubramaniam, E. L. Cayirci, « A survey on sensor networks », IEEE Communications Magazine, Vol. 40, No 8, p. 102-114, 2002.

[4] B. WARneke, M. LAst, B. Leibowitz And K. Pister, « Smart Dust : communicating with a cubic-millimeter computer », IEEE Computer, Vol. 34, $\mathrm{N}^{\circ}$ 1, p. 44-51, 2001.

[5] S. Tilak, N.B. AbU-Ghazaleh, W. Heinzelman, « A taxonomy of wireless micro-sensor network models », Mobile Computing and Communications Review, Vol. 6, $\mathrm{N}^{\mathrm{o}}$ 2, p. 28-36, 
2002.

[6] Mark A. Perillo, Wendi B. Heinzelman, « Wireless Sensor Network Protocols », Algorithms and Protocols for Wireless and Mobile Networks, Eds. A. Boukerche and al., CRC Hall Publishers, 2004.

[7] K. Sohrabi, J. Gao, V. Ailawadhi, G. J. Pottie, « Protocols for Self-Organization of a Wireless Sensor Network », IEEE personal communications, Vol. 7, $\mathrm{N}^{\circ}$ 5, p. 16-27, 2000.

[8] C. Intanagonwiwat and R. Govindan and D. Estrin, «Directed Diffusion : a scalable and robust communication paradigm for sensor networks », ACM Press, p. 56-67, 2000.

[9] B. WARnEKe, K.S.J. PISTER, « MEMS for Distributed Wireless Sensor Networks », 9th International Conference on Electronics, Circuits and Systems, Croatia, Vol. 1, p. 291-294, 2002.

[10] Saffre, Fabrice, TAteson, Richard, GHANEA-Hercock, Robert, « Reliable sensor networks using decentralised channel selection », Computer Networks, Elsevier, Vol. 46, $\mathrm{N}^{\circ}$ 5, p. 651-663, 2004.

[11] S. FAyE, J. F. Myoupo, « An Ultra Hierarchical Clustering-Based Secure Aggregation Protocol for Wireless Sensor Networks », AISS : Advances in Information Sciences and Service Sciences, Vol. 3, N 9, p. $309-319,2011$.

[12] A. Perrig, R. Szewczyk, V. Wen, D. Culler, J.D. Tygar, « SPINS : Security protocols for sensor networks », Wireless networks, Vol. 8, $\mathrm{N}^{\circ}$ 5, p. 521-534, 2002.

[13] C. Karlof, N. SaStRy And D. WAgner, « TinySec : A Link Layer Security Architecture for Wireless Sensor Networks », in : Proc. of the 2nd international conference on Embedded networked sensor systems, ACM, p. 162-175, 2004.

[14] D. Wei And S. Kaplan And H. A. Chan, « Energy Efficient Clustering Algorithms for Wireless », in : Sensor Networks, Proceedings of IEEE Conference on Communications, Beijing, IEEE, p. 236-240, 2008.

[15] Pujolle, GuY, «Les réseaux : Edition 2014 », Editions Eyrolles, 2014.

[16] Welsh, Dominic JA, Powell, Martin B, « An upper bound for the chromatic number of a graph and its application to timetabling problems », The Computer Journal, Br Computer Soc, Vol. 10, $\mathrm{N}^{\circ}$ 1, p. 85-86, 1967.

[17] BRÉLAZ, DANIEL, « New methods to color the vertices of a graph », Communications of the ACM, Vol. 22, $\mathrm{N}^{\circ}$ 4, p. 251-256, 1979.

[18] ZyKov, AleXANDER AlEKSANDROVICH, « On some properties of linear complexes », Matematicheskii sbornik, Russian Academy of Sciences, Steklov Mathematical Institute of Russian Academy of Sciences, Vol. 66, N 2, p. 163-188, 1949.

[19] Corneil, Derek G., Graham, Bruce, « An algorithm for determining the chromatic number of a graph », SIAM Journal on Computing, SIAM, Vol. 2, $\mathrm{N}^{\circ}$ 4, p. 311-318, 1973.

\section{A. Practical example for determination of priority clusters}

With the example of figure 5 , the determination of priority clusters is performed as follow :

Lists construction :

$L[0]=\emptyset ; L[1]=[(1,1),(1,2),(1,3),(1,4)] ; L[2]=[(2,1),(2,4),(2,5)]$

Extraction of clusters sublists which follow :

$L_{s}[0]=\emptyset ; L_{s}[1]=[(1,1),(1,2),(1,3),(1,4)] ; L_{s}[2]=[(2,4),(2,5)]$

The longest sub-list : $L_{s p}=L_{s}[1]=[(1,1),(1,2),(1,3),(1,4)]$

Calculation of the coordinates $(x, y)$ : 
$x=1-1=0$ and $y=$ floor $($ Average $(1,2,3,4))=$ floor $(2.5)=2$

Filling stategic clusters : The cluster $(0,2)$ is free, an actuator should be sent into it. By repeating the process : We should put an actuator (if there are available) in the cluster $(0,4) \ldots$

\section{B. Practical example of moving a mobile sensor}

Let's move an actuator from the cluster $A(1,1)$ to the cluster $B(0,3)$. For our tests, let's suppose : the scope of the sink is $30.0 \mathrm{~m}$ and the virtual architecture includes 3 coronas with $e=10.0 \mathrm{~m}$ each; there are 8 angular sectors of $\alpha=\frac{\pi}{4}$ rad each.

Polar Coordinates : $A(15.0,1178 \mathrm{rad})$ and $B(5.0,2.749 \mathrm{rad})$

Cartesian coordinates : $A(13858,5740)$ and $B(1913,4619)$

Distance : $p=\|\overrightarrow{A B}\|=15.811$

Displacement angle : $B_{x}^{\prime}=B_{x}-A_{x}=-11.945$ and $B_{y}^{\prime}=B_{y}-A_{y}=-10.359$, then $\alpha_{0}=0.856 \mathrm{rad}$. Since $B_{x}^{\prime}<0$ and $B_{y}^{\prime}<=0$ then B is in sector 2 and thus, $\varphi=\pi+\alpha_{0}=3.998 \operatorname{rad}$ or $\varphi=229.065^{\circ}$.

Conclusion : To strengthen the area $(0,3)$, the sink node asks the actuator of the cluster $(1,1)$ to cover a distance $p=15.811 \mathrm{~m}$ with an angle of $\varphi=229,065^{\circ}$.

\section{Algorithms}

\section{Lexicon of parameters and functions}




\begin{tabular}{|c|c|}
\hline Parameters & Description \\
\hline numberofneighbours & $\begin{array}{l}\text { Numbers of neighbors in a cluster including those at } \\
\text { two hops }\end{array}$ \\
\hline NumberOfFreq & $\begin{array}{l}\text { Contains the number of channels we need in our net- } \\
\text { work. The frequency range will then be divided by this } \\
\text { number }\end{array}$ \\
\hline$V_{c 2}$ & Neighbors of cluster 'c' including those at two hops \\
\hline NeighbouringFreqOfC & $\begin{array}{l}\text { Set of all the indices of the channels used in the vicinity } \\
\text { of a cluster }\end{array}$ \\
\hline neighbouringFreqs $(L)$ & $\begin{array}{l}\text { Function that returns the indexes of the channels used } \\
\text { by the clusters passed in parameter (L) }\end{array}$ \\
\hline UsableFreqForC & Indexes of unused channels in the vicinity of a cluster \\
\hline cluster.freq & Denotes the index of the channel used by this cluster \\
\hline NumberOfFreq.size() & Returns the current number of channels required \\
\hline first $(L)$ & Returns the first item in list $\mathrm{L}$ \\
\hline amplitude & Amplitude of a channel \\
\hline Stop_freq & End of the range of frequencies allocated to the network \\
\hline Start_freq & $\begin{array}{l}\text { Start of the range of frequencies allocated to the net- } \\
\text { work }\end{array}$ \\
\hline neighbourOf(cluster) & $\begin{array}{l}\text { Function that returns the neighbors of the cluster passed } \\
\text { in parameter }\end{array}$ \\
\hline $\begin{array}{l}\text { UseFreq(amplitude, freq, } \\
\text { neighbourFreqs, dest })\end{array}$ & $\begin{array}{l}\text { Message that allows to communicate to a cluster the } \\
\text { index of the channel on which to communicate ( freq), } \\
\text { as well as the indexes of the channels of its neighbors } \\
\text { (neighbourFreqs). amplitude denotes the amplitude } \\
\text { of the channel, (dest) is the destination cluster. }\end{array}$ \\
\hline$m s g()$ & Function that allows to build several types of messages \\
\hline Radio.tansmit $\left(r_{i}, f_{0}\right.$, UseFreq $)$ & $\begin{array}{l}\text { Function that allows the sink to broadcast a message } \\
\text { (for example } U \text { seFreq) on a channel (for example, on } \\
\text { the channel of index } f_{0} \text { ) and within a radius }\left(r_{i}\right)\end{array}$ \\
\hline
\end{tabular}




\begin{tabular}{|c|c|}
\hline Parameters & Description \\
\hline Radio.tansmit(message, freq) & $\begin{array}{l}\text { Function which allows a sensor to broadcast a message } \\
\text { on a channel of indexe freq. The coverage radius is that } \\
\text { of the sensor. }\end{array}$ \\
\hline UseFreq.dest & $\begin{array}{l}\text { Allows to extract the coordinates of the recipient of a } \\
\text { message }\end{array}$ \\
\hline UseFreq.neighbourFreqs & Denote frequency indexes used by neighboring clusters \\
\hline numberOfHops & Number of hops \\
\hline Detect & $\begin{array}{l}\text { Message allowing different clusters to identify them- } \\
\text { selves }\end{array}$ \\
\hline EndTime & End date of the empty cluster detection algorithm \\
\hline$A K C$ & Message acting as acknowledgment of receipt \\
\hline relay_cluster & $\begin{array}{l}\text { Cluster through which the } \mathrm{CH} \text { sends messages to the } \\
\text { sink }\end{array}$ \\
\hline $\operatorname{relay}(x, y, z)$ & $\begin{array}{l}\text { Table that allows the sink to have the coordinates of the } \\
\text { relay cluster of the cluster of coordinates }(x, y, z)\end{array}$ \\
\hline$h(x, y, z)$ & $\begin{array}{l}\text { Allows the sink to know whether the cluster of coordi- } \\
\text { nates }(x, y, z) \text { is empty or not }\end{array}$ \\
\hline are_neighbours $\left(c_{1}, c_{2}\right)$ & $\begin{array}{l}\text { Function which makes it possible to know if two clus- } \\
\text { ters } c_{1} \text { and } c_{2} \text { are neighbors or not }\end{array}$ \\
\hline$\tau$ & The duration of a slot \\
\hline$E_{r}$ & Residual energy of a sensor \\
\hline$E_{s}$ & Threshold energy of a sensor \\
\hline Detect_timerNotReceived & $\begin{array}{l}\text { Boolean that lets you know if a sensor has received a } \\
\text { Detect_timer }\end{array}$ \\
\hline time() & Function that returns the local time of a sensor \\
\hline Detect_timer & $\begin{array}{l}\text { Message that indicates to the sensors the date on which } \\
\text { the } \mathrm{CH} \text { will be elected }\end{array}$ \\
\hline fork_launch_CH_election & $\begin{array}{l}\text { Sub-process that effectively replaces an active wait } \\
\text { that consumes enough energy. The latter schedules the } \\
\text { launch of the } \mathrm{CH} \text { election. }\end{array}$ \\
\hline fork_send_ACK & $\begin{array}{l}\text { Sub-process that effectively replaces an active wait that } \\
\text { consumes enough energy. The latter schedules the sen- } \\
\text { ding of an acknowledgment of receipt. }\end{array}$ \\
\hline $\operatorname{node}(I D)$ & Returns a sensor from its $I D$ \\
\hline Head1 & $\begin{array}{l}\text { Message used during the election of the } \mathrm{CH} \text { to choose } \\
\text { the sensor closest to the sink in terms of number of } \\
\text { hops. }\end{array}$ \\
\hline Head2 & $\begin{array}{l}\text { Message used during the election of the } \mathrm{CH} \text { to choose } \\
\text { the } \mathrm{CH} \text { among the sensors closest to the sink in terms of } \\
\text { number of hops and having the greatest residual energy } \\
\left(E_{r}\right) \text {. }\end{array}$ \\
\hline Head1MessageNotReceived & $\begin{array}{l}\text { Boolean which allows to know if a sensor has pre- } \\
\text { viously received a Head } 1 \text { message. }\end{array}$ \\
\hline Head2MessageNotReceived & $\begin{array}{l}\text { Boolean which allows to know if a sensor has pre- } \\
\text { viously received a Head } 2 \text { message. }\end{array}$ \\
\hline cluster_head & Local reference of the sensor playing the role of $\mathrm{CH}$. \\
\hline
\end{tabular}



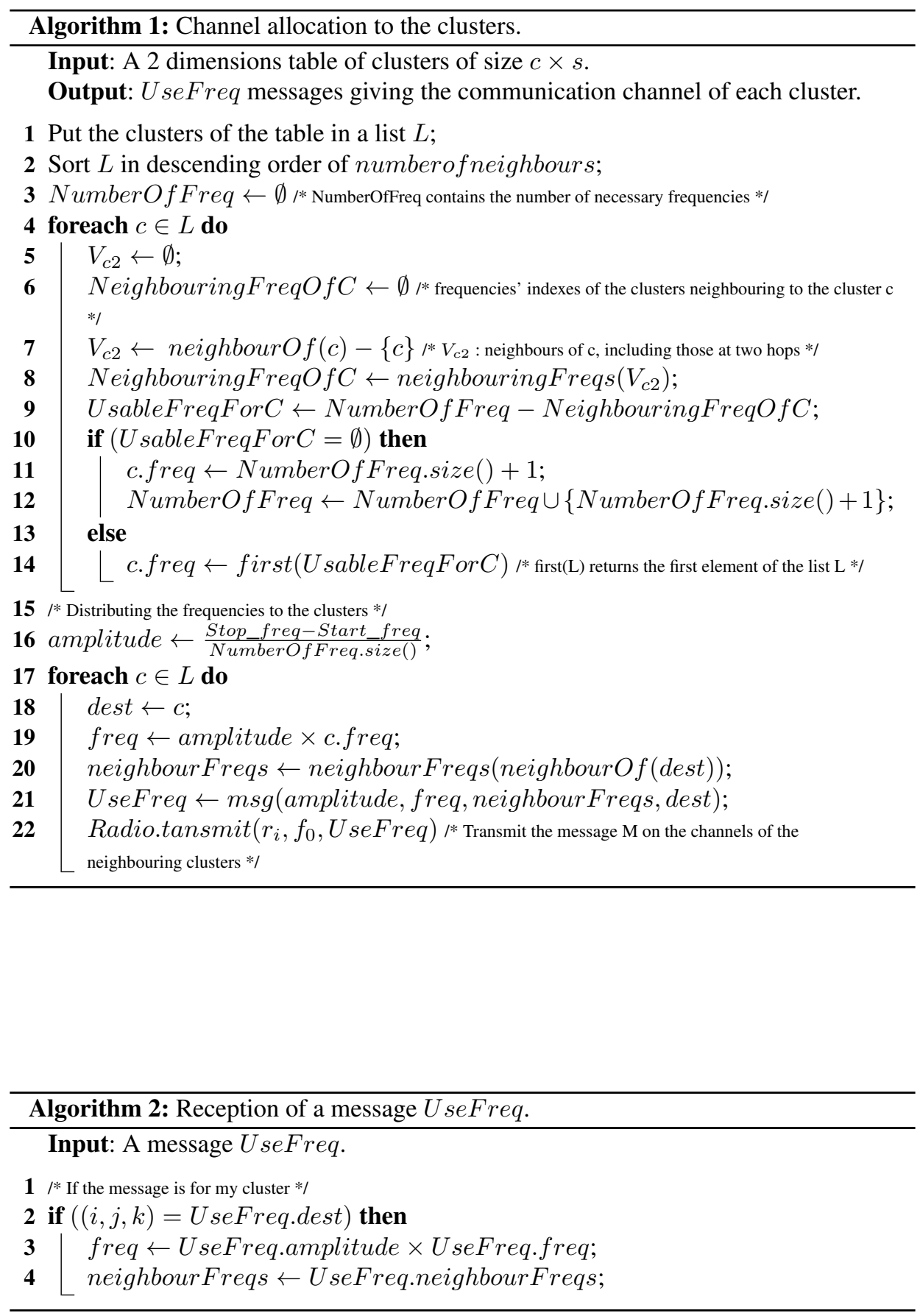


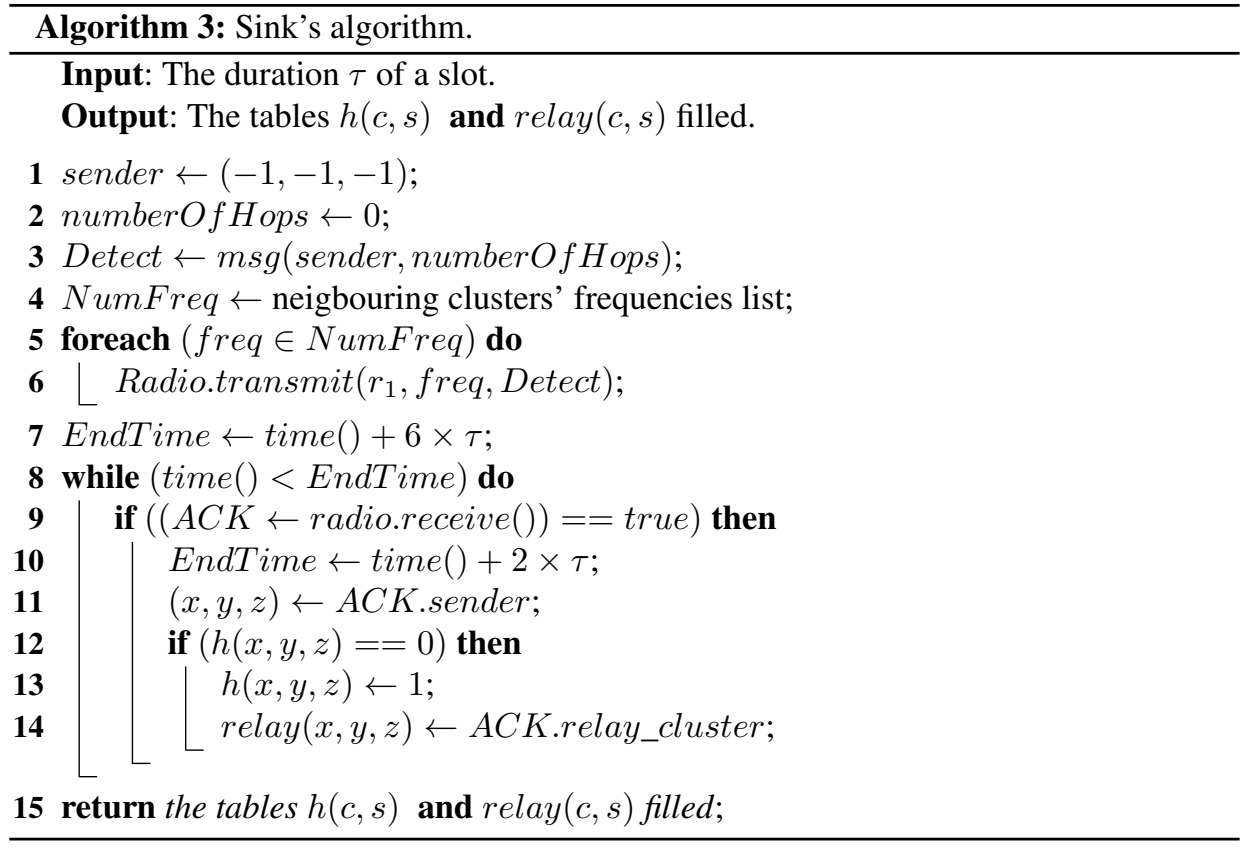



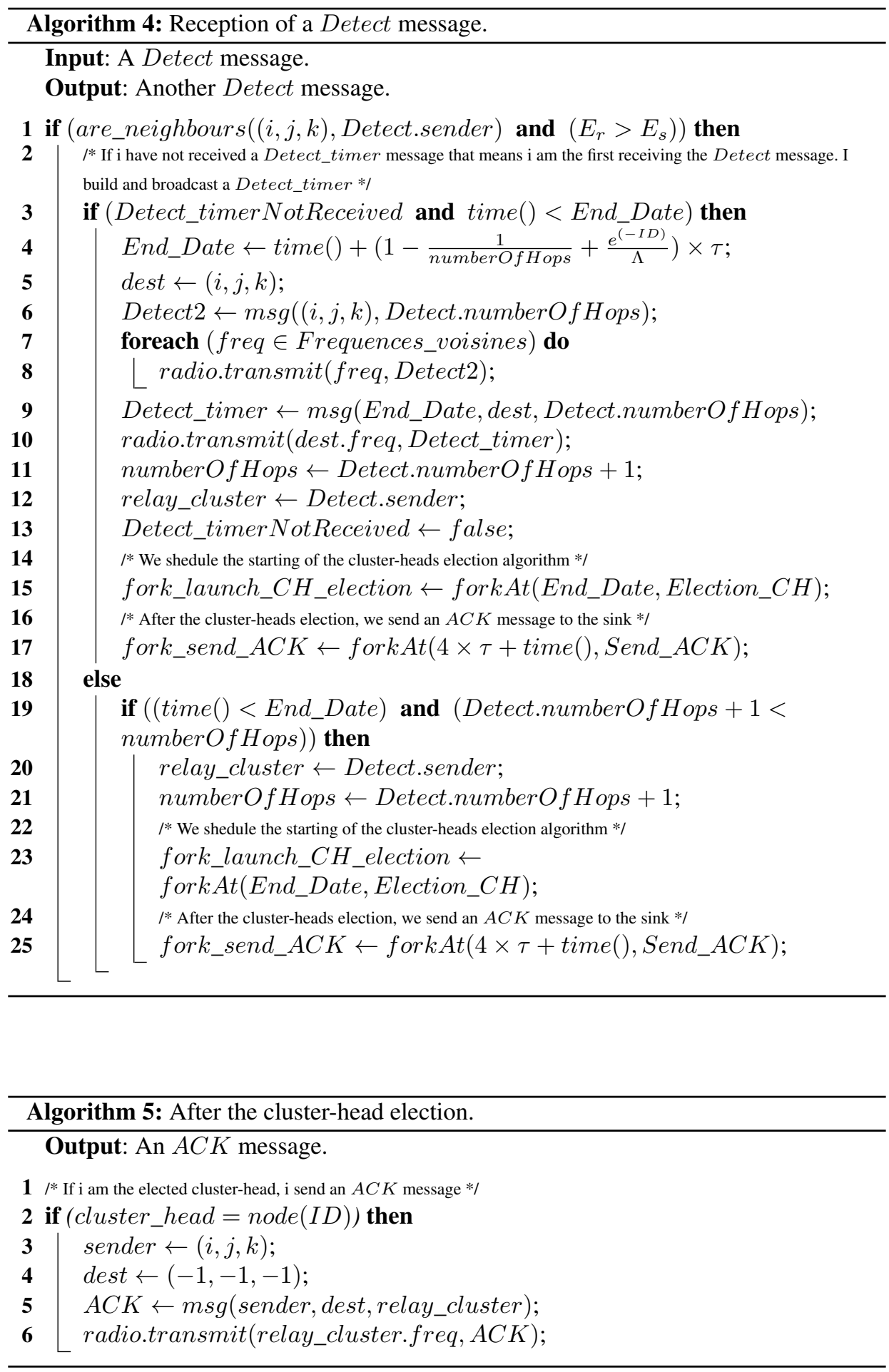

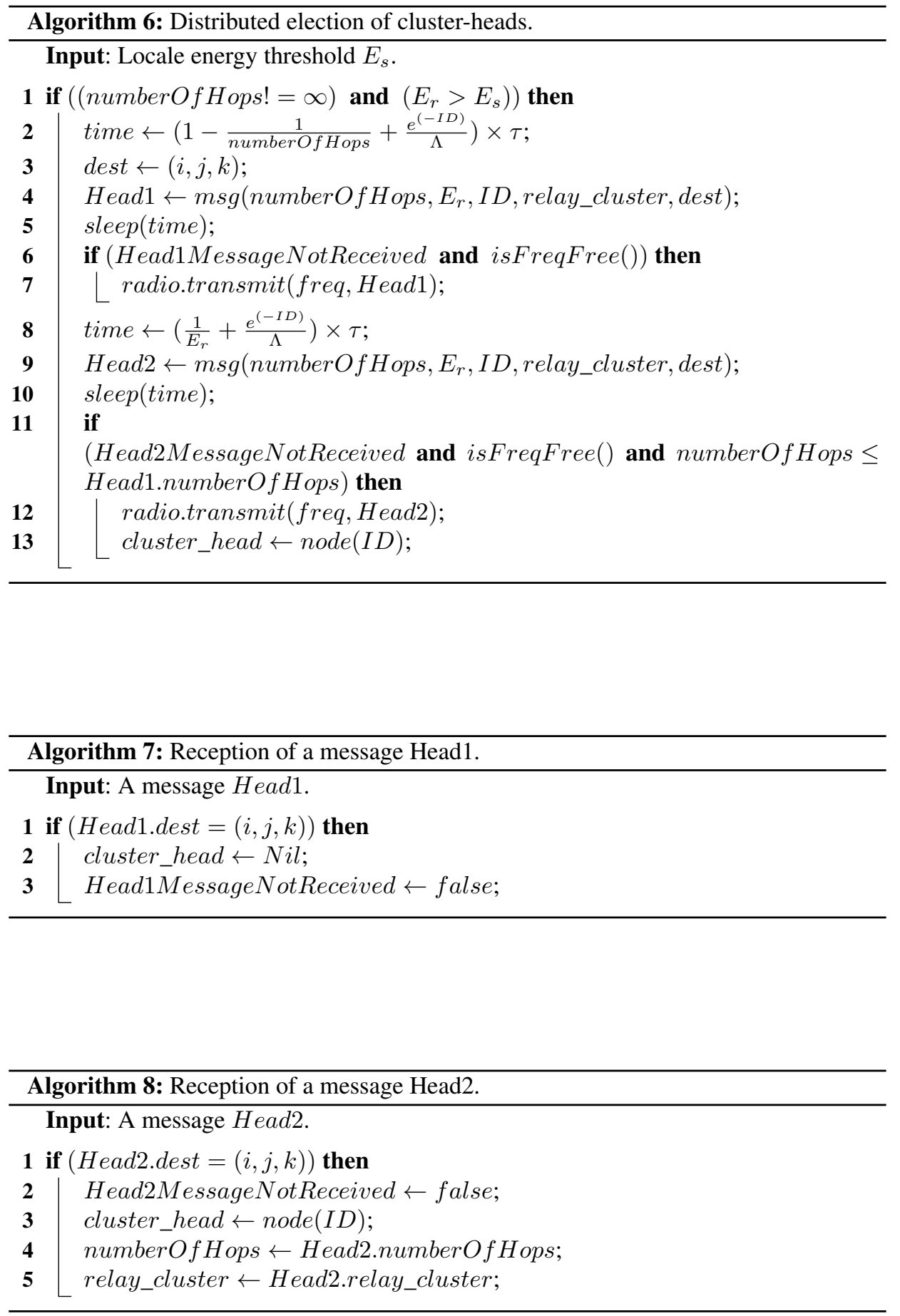